\title{
A Particulate Isotopic Standard of Uranium and Plutonium in an Aluminosilicate Matrix
}

\author{
J. J. Stoffel(s) and J. K. Briant \\ Pacific Northwest Laboratory, Richland, Washington, USA \\ D. S. Simons \\ National Institute of Standards and Technology, Gaithersburg, Maryland, USA
}

\begin{abstract}
Mixed-actinide microstandard particles have been produced for calibration and performance testing of isotope-ratio mass spectrometers and ion and electron microprobe instruments. The spherical micrometer-size particles consist of an aluminosilicate matrix loaded with $2.2 \%$ by weight of isotopically certified uranium and $0.11 \%$ by weight of isotopically certified plutonium. The uranium and plutonium isotopic compositions have been verified by both thermal ionization mass spectrometry and secondary ionization mass spectrometry (SIMS). The elemental composition of the microspheres has been determined by both electron microprobe and SIMS analysis. (J Am Soc Mass Spectrom 1994, 5, 852-858)
\end{abstract}

A t the Pacific Northwest Laboratory (PNL) and the National Institute of Standards and Technology (NIST), we measure trace levels of uranium, plutonium, and other artificial radionuclides in environmental samples by thermal ionization mass spectrometry (TIMS) and secondary ionization mass spectrometry (SMS). The isotopic analysis of individual actinide-bearing particles can be used to associate these radionuclides with specific sources of environmental contamination. For analysis of such particles, we calibrate our instruments with particulate isotopic standards to maintain the accuracy of analytical results. Several particle standards of uranium have been available for this purpose [1]. PNL recently produced a plutonium particle standard for this purpose that consists of spherical micrometer-size aluminosilicate spheres loaded with a minor concentration of isotopically certified plutonium [2]. That work has now been extended by producing a new particle standard that consists of aluminosilicate spheres loaded with minor concentrations of both isotopically certified uranium and plutonium.

\section{Particle Standard Production}

The same facilities and methods that were used to produce the plutonium particle standard [2] were applied to the production of the mixed-actinide standard. In brief, the particle production process involved charging a colloidal suspension of high-purity aluminosilicate clay (Inhalation Toxicology Research Insti-

Address reprint requests to Jim Stoffel(s), Pacific Northwest Laboratory, P.O. Box 999, MS P7-07, Richland, WA 99352-0999. tute, Lovelace Biomedical and Environmental Research Institute, Albuquerque, $\mathrm{NM})^{*}$ with uranium and plutonium by ion exchange, generation and fusing of aerosol particles from the U-Pu-labeled clay suspension [3, 4], and size separation of the generated particles in a centrifugal aerosol spectrometer [5]. As previously, these operations were conducted in glove boxes equipped to prevent the release of particles to the workplace or the environment.

The same certified plutonium isotopic standard material, CRM 138 (U.S. Department of Energy, New Brunswick Laboratory, Argonne, IL), that was used for the plutonium particle standard was used for this mixed-actinide standard. The certified uranium isotopic standard material used was the original NBS U005 (National Bureau of Standards, now National Institute of Standards and Technology, Gaithersburg, MD). The isotopic compositions of these two standard materials are given in Table 1.

The target concentrations (weight percent) in the aluminosilicate matrix were $2 \%$ for the uranium and $0.04 \%$ for the plutonium. The plutonium concentration was chosen to give, in a thermal ionization mass spectrometer, an ion count rate one-tenth of the uranium ion count rate, with $\mathrm{Pu}$ ionization efficiency five times that of $\mathrm{U}$.

We considered two procedures for charging the clay particles with the two actinide elements in the desired proportion. One possible procedure was to charge two separate aliquots of clay, one with uranium and one

* Certain commercial equipment, instruments, and materials are identified in this paper to specify adequately the experimental procedure. Such identification does not imply recommendation or endorsement by PNL or NIST, nor does it imply that the equipment or materials are necessarily the best available for the purpose. 
Table 1. Compositions of the uranium isotopic standard NBS U005 and the plutonium isotopic standard CRM 138

\begin{tabular}{lcc} 
Isotope & $\begin{array}{c}\text { NBS U005 } \\
\text { atom \% U } \\
\pm 95 \% \mathrm{CL}^{\mathrm{b}} \text { error }\end{array}$ & $\begin{array}{c}\text { CRM 138 } \\
\text { atom \% Pu } \\
\pm 95 \% \mathrm{CL}^{\mathrm{b}} \text { error }\end{array}$ \\
\hline \hline 234 & $0.00218 \pm 0.00004$ & \\
235 & $0.4895 \pm 0.0005$ & \\
236 & $0.00466 \pm 0.00005$ & \\
238 & $99.504 \pm 0.001$ & $0.010 \pm 0.001$ \\
239 & & $91.855 \pm 0.010$ \\
240 & & $7.926 \pm 0.010$ \\
241 & & $0.176 \pm 0.001$ \\
242 & & $0.0330 \pm 0.0003$ \\
\hline
\end{tabular}

"Plutonium values are corrected for radioactive decay to 1 January 1993.

Confidence level.

with plutonium, and then mix the two clay suspensions together to give the desired U:Pu ratio. A potential problem with this procedure was that the U:Pu ratio might not be homogeneous from particle to particle. Because the colloidal clay micelles are a relatively large $0.2 \mu \mathrm{m}$ [3], the number of Pu-loaded micelles in the final aggregate spheres would be small $(\sim 20)$. The resulting particle-to-particle variability in plutonium concentration would be $\sim 20 \%$ on a purely statistical basis, assuming a Poisson distribution of micelles.

Another approach for charging the clay with two actinides was to mix them in the desired proportion in solution and use the mixture to charge a single aliquot of the colloidal clay suspension. The problem with this approach was that the relative loading efficiency for the two actinides in a mixture was unknown. The U:Pu ratio in the clay micelles could be quite different from that of the solution. However, because this was the simpler of the two procedures to test, we decided to pursue it.

Three clay charging tests were conducted with chloride solutions that had U:Pu ratios of 1:1, 10:1, and 20:1. For each test, the mixed-actinide solution was added to an aliquot of the colloidal clay suspension and stirred for $2 \mathrm{~h}$. The clay was then filtered, washed, and resuspended in water. A small amount of the suspension was dried on a beryllium mount for wavelength-dispersive $x$-ray analysis on a JEOL JXA-8600 electron microprobe (JEOL U.S.A., Peabody, MA). Results of the analyses are given in Table 2.

Discrepancies are apparent in Table 2 between the calculated maximum actinide concentrations based on complete charging into the clay and the actual measured concentrations. Some differences are greater than can be accounted for by normal gravimetric and volumetric errors or by errors in the electron probe analysis. We believe that the discrepancies arose because our calculations treated the clay suspensions as perfect colloids, whereas they are not. Subsequent investigation has shown that the clay particles settle slowly, thereby producing a concentration gradient in the sus-
Table 2. Results of electron probe analysis of clay charged with uranium and plutonium from mixed solutions

\begin{tabular}{|c|c|c|c|}
\hline \multirow[b]{2}{*}{$U$ and $P u$ in clay } & \multicolumn{3}{|c|}{$\begin{array}{l}\text { U:Pu weight ratio } \\
\text { in solution }\end{array}$} \\
\hline & 1 & 10 & 20 \\
\hline U wt \% (calculated maximum) & 0.20 & 2.0 & 3.9 \\
\hline U wt $\%$ (measured) $^{a}$ & 0.26 & 2.9 & 4.9 \\
\hline Pu wt \% (calculated maximum) & 0.20 & 0.20 & 0.20 \\
\hline Pu wt \% (measured) $)^{\nexists}$ & 0.20 & 0.27 & 0.10 \\
\hline
\end{tabular}

The estimated accuracy is $\leq 10 \%$ for elements present at $\geq 1 \%$ and $\pm 0.1 \mathrm{wt} \%$ for elements present at $<1 \%$. For a discussion of discrepancies, refer to the text.

pensions. Our experimental procedure did not include steps to avoid the effects of sedimentation. If an aliquot were drawn off near the surface of the suspensions where the clay concentration decreases with time, the maximum relative actinide concentration in the charged clay would be increased.

In all three clay charging tests, the measured uranium weight percent exceeded the calculated maximum based on the makeup concentration of the clay suspension. This indicates that sedimentation was a consistent factor in the clay charging tests. It also indicates that all of the uranium available in solution was probably charged into the clay. The situation with respect to the plutonium is less clear because the measurement uncertainties are considerably greater percentagewise at the lower plutonium concentration.

On the basis of the test results, we used a solution with a U:Pu ratio of $10: 1$ to charge clay for the particle production run. Five milliliters of the clay suspension were nebulized with a Lovelace nebulizer (Lovelace Foundation, Albuquerque, NM). The aerosol droplets were passed through a tube furnace at $11500^{\circ} \mathrm{C}$, where they fused into solid particles $[3,4]$. The polydisperse actinide-loaded particles proceeded into a centrifugal aerosol spectrometer where they were deposited onto a long foil according to their aerodynamic equivalent diameter [5]. The foil was separated into segments, each of which held particles close to the same size. Two foil segments, which were known from the operating parameters of the aerosol spectrometer to hold particles close to $2-\mu \mathrm{m}$ diameter, were selected for further processing. The particles on these segments were resuspended in separate aliquots of ethanol. Dilute collodion was added and the particles were dispersed in a collodion layer on polycarbonate microscope slides. Individual particles from the two size lots were isolated from the slide mounts for characterization.

\section{Particle Standard Characterization}

\section{Morphology}

As reported previously, the heat treatment of the particles produces an amorphous structure [2]. The shape 
and size of particles produced were measured with a JEOL Model 840 scanning electron microscope equipped with a computerized image analysis system (JEOL U.S.A., Peabody, MA). Prior to measurement of the U-Pu-clay particles, the system was calibrated with National Bureau of Standards (NBS) traceable standard polystyrene latex spheres of known diameter.

The particles are near spherical, as can be seen in Figure 1. For lot 1, an average shape factor (ratio of maximum to minimum diameter) of 1.065 was determined from measurements on 48 discrete particles. Size measurements on the same particles gave an average diameter of $1.95 \mu \mathrm{m}$. The distribution of particle sizes, shown in Figure 2, has a standard deviation of $\pm 0.22 \mu \mathrm{m}$.

Lot 2 has an average shape factor of 1.054 and an average diameter of $2.16 \mu \mathrm{m}$. The size distribution (Figure 2) has a standard deviation of $\pm 0.21 \mu \mathrm{m}$.

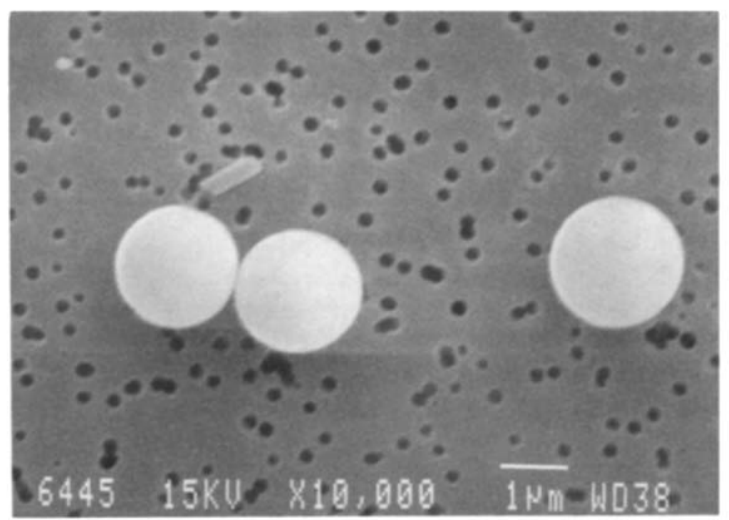

Figure 1. SEM photograph of U-Pu-clay microspheres.

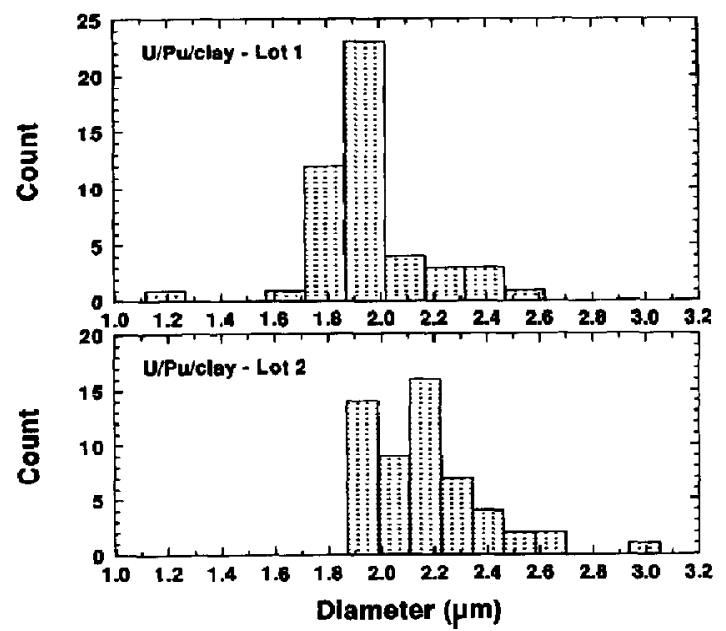

Figure 2. Size distributions of $\mathrm{U}-\mathbf{P u}-$ clay microspheres from lot 1 and lot 2 .
These parameters were determined from measurements on 55 discrete particles.

Users of the previous $\mathrm{Pu}$-clay microstandard questioned the reported size of $1.75 \mu \mathrm{m}$. We found that the original size measurements were in error due to the use of inadequate magnification. Therefore, we individually sized 10 more of those microspheres by scanning electron microscopy (SEM). The correct average diameter and standard deviation are $2.09 \pm 0.16 \mu \mathrm{m}$.

\section{Elemental Composition}

The elemental composition of the U-Pu-clay microspheres was determined by wavelength-dispersive $x$ ray analysis on the JEOL JXA- 8600 electron microprobe. The instrument was calibrated with standards for all the elements measured. The standards were flat, carbon-coated surfaces of the element or a stoichiometric compound.

Six individually mounted spheres, three from each size lot, were analyzed three times each. There was no discernible difference in composition of the two lots. The average composition is given in Table 3. The estimated accuracy is better than $10 \%$ for elements present at $\geq 1 \%$, as determined by measurements on NBS glass-particle standards of similar composition and size.

The composition of the aluminosilicate matrix shows some differences from that of the previous $\mathrm{Pu}$-clay standard. Sodium, which was present at a concentration of $0.2-0.3 \%$ in the previous standard, is an order of magnitude lower in concentration in the new standard. The magnesium concentration is two-thirds of that in the Pu-clay standard. The sodium may have been replaced more completely in the ion-exchange process by which the uranium and plutonium were loaded into the matrix, or it may have been washed more completely from the actinide-loaded clay. The magnesium is not affected by these processes. It is possible that some loss of both elements may have occurred in the particle fusing process because the

Table 3. Elemental composition of six U-Pu-clay microspheres by electron probe analysis

\begin{tabular}{lc}
\hline Element & $\begin{array}{c}\text { Average } \\
\text { weight percent }\end{array}$ \\
\hline \hline $\mathrm{Na}$ & 0.01 \\
$\mathrm{Mg}$ & 3.4 \\
$\mathrm{Al}$ & 11.1 \\
$\mathrm{Si}$ & 32.2 \\
$\mathrm{Ca}$ & 0.6 \\
$\mathrm{Fe}$ & 0.8 \\
$\mathrm{U}$ & 2.2 \\
$\mathrm{Pu}$ & 0.11 \\
$\mathrm{O}$ & Balance \\
\hline
\end{tabular}

a The estimated accuracy. as determined by measurements on NBS glass-particle standards of similar composition and size, is $\leq 10 \%$ for elements present at $\geq 1 \%$ and \pm 0.1 wt $\%$ for elements present at $<1 \%$ 
fusing temperature exceeded the boiling points of sodium and magnesium.

Further detail on minor elemental constituents is given below in the section on secondary ionization mass spectrometry. Other physical parameters, calculated from the measured sizes and elemental composition, are given in Table 4.

\section{Isotopic Composition}

In addition to the intentionally loaded NBS U005, the $\mathrm{U}-\mathrm{Pu}$-clay microspheres have a minor contribution of uranium from the decay of the plutonium isotopic standard material. The following values were calculated for the decay uranium concentration in CRM 138 as of 1 January 1993, 30.5 years after the original chemical purification [6]:

$$
\begin{aligned}
& { }^{234} \mathrm{U}:{ }^{239} \mathrm{Pu}=2.97 \times 10^{-5} \\
& { }^{235} \mathrm{U}:{ }^{239} \mathrm{Pu}=8.77 \times 10^{-4} \\
& { }^{236} \mathrm{U}:{ }^{239} \mathrm{Pu}=2.78 \times 10^{-4} \\
& { }^{238} \mathrm{U}:{ }^{239} \mathrm{Pu}=2.02 \times 10^{-8}
\end{aligned}
$$

The measured uranium concentration in the matrix of the mixed-actinide standard microspheres is $2.2 \%$ and the plutonium concentration is $0.11 \%$. Because the plutonium is loaded into the clay at a concentration of 0.050 relative to that of the uranium, the ratios of the decay uranium isotopes to the ${ }^{238} \mathrm{U}$ in the U005 standard are

$$
\begin{aligned}
& { }^{234} \mathrm{U}:{ }^{238} \mathrm{U}=1.37 \times 10^{-6}=1.4 \text {-ppm excess } \\
& { }^{235} \mathrm{U}:{ }^{238} \mathrm{U}=4.05 \times 10^{-5}=40.5 \text {-ppm excess } \\
& { }^{236} \mathrm{U:}{ }^{238} \mathrm{U}=1.28 \times 10^{-5}=12.8 \text {-ppm excess } \\
& { }^{238} \mathrm{U}:{ }^{238} \mathrm{U}=9.3 \times 10^{-10}=0.0 \text {-ppm excess }
\end{aligned}
$$

The further contribution from natural uranium in the clay matrix was previously determined to be negligible

Table 4. Physical parameters of the U-Pu-clay standard microspheres

\begin{tabular}{lcc}
\hline Average particle & Lot 1 & Lot 2 \\
\hline \hline Diameter $(\mu \mathrm{m})$ & 1.95 & 2.16 \\
Volume $\left(\mu \mathrm{m}^{3}\right)$ & 3.88 & 5.28 \\
Density $\left(\mathrm{pg} / \mu \mathrm{m}^{3}\right)$ & 2.2 & 2.2 \\
Weight $(\mathrm{pg})$ & 8.5 & 11.6 \\
U weight $(\mathrm{fg})$ & 188. & 255. \\
U atoms $\left(10^{8}\right)$ & 4.8 & 6.5 \\
Pu weight $(\mathrm{fg})$ & 9.4 & 12.8 \\
Pu atoms $\left(10^{7}\right)$ & 2.4 & 3.2 \\
Activity $(\mathrm{fCi})$ & 2.6 & 3.6 \\
\hline
\end{tabular}

\footnotetext{
${ }^{a}$ Calculated activity includes $\alpha$ decay of ${ }^{238} \mathrm{Pu},{ }^{239} \mathrm{Pu}$, and ${ }^{240} \mathrm{Pu}$.
} Calculated activity includes $\alpha$ decay of
$\beta$ decay of ${ }^{241} \mathrm{Pu}$, and $\alpha$ decay of ${ }^{241} \mathrm{Am}$ by ion-microprobe (SIMS) measurements on the Pu-clay microspheres [7]. The uranium isotopic composition of the U-Pu-clay microspheres, as of 1 January 1993, is then

\begin{tabular}{cc} 
Isotope & Atom $\% \mathrm{U}$ \\
\hline 234 & 0.0023 \\
235 & 0.4935 \\
236 & 0.0059 \\
238 & 99.498
\end{tabular}

Americium-241 is also introduced by the radioactive decay of ${ }^{241} \mathrm{Pu}$ in the plutonium isotopic standard. Based on an effective americium separation date of 6 July 1962 [6] and including the decay of americium itself, we expect as of 1 January 1993,

$$
{ }^{241} \mathrm{Am}:{ }^{241} \mathrm{Pu}=3.3
$$

Thus, there should be more than three times as much ${ }^{241} \mathrm{Am}$ as ${ }^{241} \mathrm{Pu}$ in the microspheres, assuming equal efficiency of loading into the clay matrix.

Thermal Ionization Mass Spectrometry. Isotopic analysis of 16 individual micraspheres was performed on one of the PNL triple-sector thermal ionization mass spectrometers [8]. Each sphere was mounted on a single rhenium V-filament carburized at $1600^{\circ} \mathrm{C}$ and run in the mass spectrometer until completely consumed. TIMS measurement efficiency (ions detected per atoms loaded) was determined for 10 spheres, 5 spheres from each size lot, that were individually sized by SEM measurement. The total ion counts of ${ }^{238} \mathrm{U}$ and ${ }^{239} \mathrm{Pu}$ accumulated during the TIMS measurements were corrected for duty cycle factors and isotopic abundances. Ion detection efficiency was virtually $100 \%$ with the ion detector used [8].

The uranium measurement efficiency was $0.83 \pm$ $0.27 \%$ ( $1 \sigma$ standard deviation). The plutonium measurement efficiency was $4.8 \pm 1.6 \%$, which is consistent with efficiency values for the $\mathrm{Pu}$-clay microspheres [2] and for plutonium in resin beads [9] meastured on the same instrument. The Pu: $\mathrm{U}$ relative sensitivity factor of 5.7 is due to the lower ionization potential of Pu.

The analyses generally yielded the expected isotopic compositions within measurement error (see Figures 3 and 4). Except for ${ }^{238} \mathrm{Pu}$, which was below the measurement limit, minor isotopes were measurable even when they produced ion count rates of only $0.1 \mathrm{~s}^{-1}$. Measurement precision on the minor isotopes reflects the small amount of data obtainable. The number of atoms of ${ }^{234} \mathrm{U},{ }^{236} \mathrm{U}$, and ${ }^{242} \mathrm{Pu}$ in a single microsphere ranges from 8000 to 40,000 over the two size lots. The ion-count rate averages and standard deviations for 


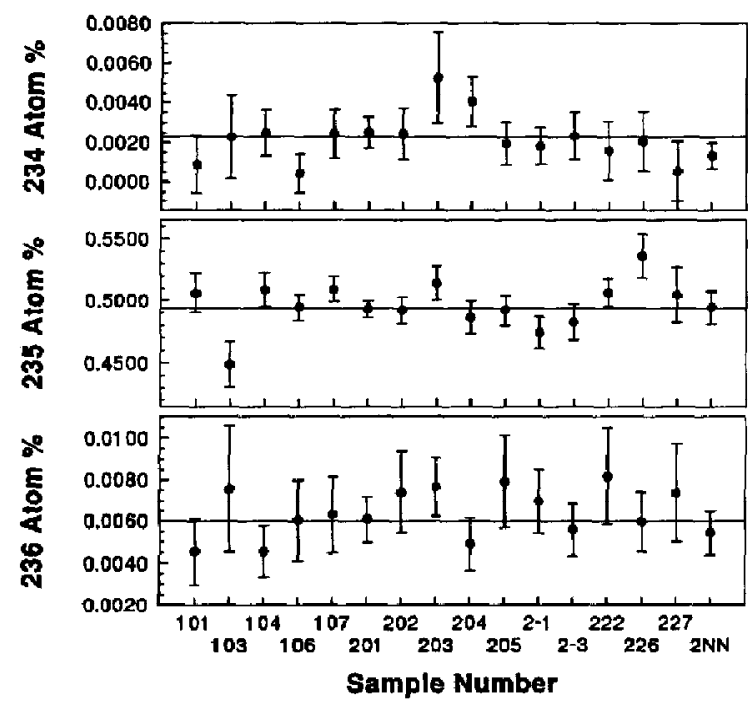

Figure 3. TTMS isotopic analysis of the uranium in $16 \mathrm{U}-\mathrm{Pu}$-clay microspheres. Solid lines represent the reference values. Error bars are the standard deviation of the mean $\left(1 \sigma_{\mathrm{m}}\right)$.

the minor isotopes were

$$
\begin{aligned}
{ }^{234} \mathrm{U} & =0.08 \pm 0.06 \mathrm{~s}^{-1} \\
{ }^{235} \mathrm{U} & =17 . \pm 8 . \mathrm{s}^{-1} \\
{ }^{236} \mathrm{U} & =0.2 \pm 0.1 \mathrm{~s}^{-1} \\
{ }^{240} \mathrm{Pu} & =116 . \pm 64 . \mathrm{s}^{-1} \\
{ }^{241} \mathrm{Pu} & =3.0 \pm 1.3 \mathrm{~s}^{-1} \\
{ }^{242} \mathrm{Pu} & =0.6 \pm 0.5 \mathrm{~s}^{-1}
\end{aligned}
$$

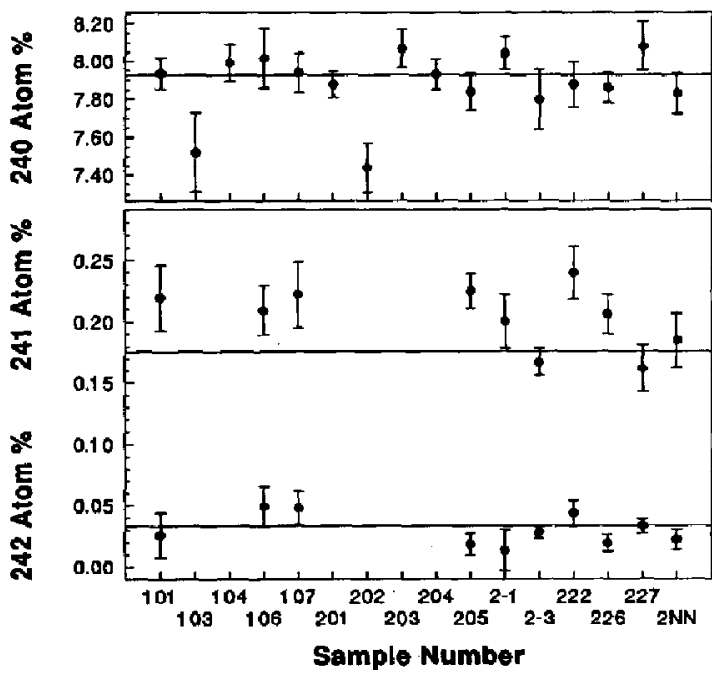

Figure 4. TIMS isotopic analysis of the plutonium in 16 U-Pu-clay microspheres. Solid lines represent the reference values. Error bars are the standard deviation of the mean $\left(1 \sigma_{\mathrm{m}}\right)$.
As with the $\mathrm{Pu}$-clay microspheres, americium evaporated prior to the onset of plutonium ion emission. Some residual americium appears to have remained during the plutonium analysis because the ${ }^{241} \mathrm{Pu}$ values are slightly elevated (Figure 4 ). The excess count rate at mass 241 averaged $0.3 \mathrm{~s}^{-1}$.

We did experience a significant molecular ion background on six samples that prevented the measurement of both ${ }^{241} \mathrm{Pu}$ and ${ }^{242} \mathrm{Pu}$. The source of contamination was not identified. We were not able to reduce the interfering background by turning off the ionizing filament for several minutes and then continuing the analysis. This standard operating procedure often significantly reduces molecular backgrounds in the actinide mass region. The mechanism by which this occurs is unknown.

Secondary Ionization Mass Spectrometry. Microspheres from lot 2 also were analyzed in a Cameca IMS-4F secondary ionization microanalyzer (Cameca Instruments, Stamford, CT). The spheres were dispersed on a rectangular piece of a Fullam "Grade $A$ " carbon planchet (Ernest F. Fullam, Latham, NY), held by a thin layer of collodion. From $\alpha$ counting of the planchet and the calculated activity from a single sphere, it was estimated that about 50 spheres were on the carbon. The spheres could not be seen with a reflected light microscope because of the relatively rough surface of the carbon.

The spheres were located in the SIMS instrument by using an automated search routine to find areas with high uranium signals. This procedure was done at low average current density so little of the sphere material was sputtered away. About 20 spheres were located in less than $1 \mathrm{~h}$.

A primary beam of $\mathrm{O}_{2}^{+}$was used for the SIMS measurements at an impact energy of $10.5 \mathrm{keV}$ and a current of 5-25 $\mathrm{nA}$, depending on the type of analysis. The beam diameter was several micrometers. It was raster-scanned over a $25-\times 25-\mu \mathrm{m}$ area with the microsphere in the center. The secondary spectrometer was set for maximum transmission by using the $25-\mu \mathrm{m}$ transfer optics setting, a contrast diaphragm of $150 \mu \mathrm{m}$ diameter, a field aperture of $1.8 \mathrm{~mm}$, and all slits fully opened.

A full mass spectrum from a single sphere is shown in Figure 5. The spectrum was taken at $1 \mathrm{~s} / \mathrm{u}$ in a peak-hopping mode with a primary beam current of 10 $\mathrm{nA}$ and a sample voltage offset of $10 \mathrm{~V}$ to provide some discrimination against molecular ions. The spectrum shows a number of minor elemental constituents that were not detected by the electron probe analysis; it is very similar to the spectrum of the $\mathrm{Pu}$-clay microsphere (see ref 7 ). The main differences are the presence of $U$ peaks and the absence of $\mathrm{Cu}$. The $\mathrm{Cu}$ in the $\mathbf{P u}$-clay spectrum may have been an instrumental background contaminant.

A partial mass spectrum that covers the actinide mass range is shown in Figure 6. The spectrum, again 


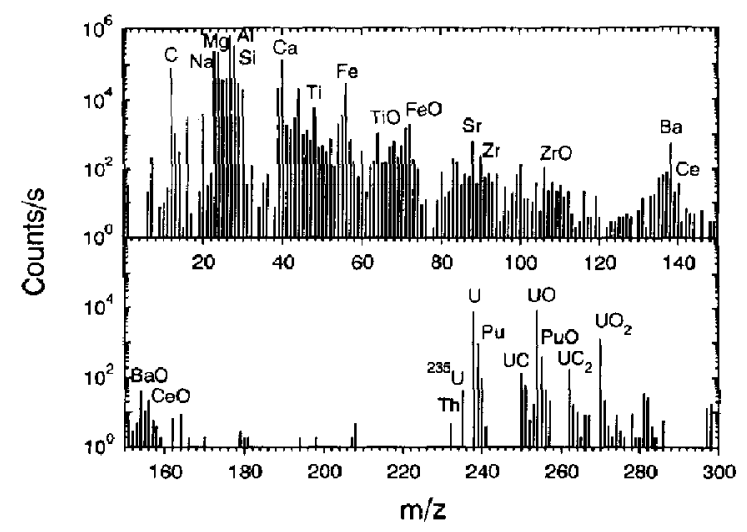

Figure 5. SIMS full mass spectrum from a single U-Pu-clay microsphere.

from a single sphere, was taken continuously at 1 $s /$ chanmel and 10 channels/ $u$. The beam current was $25 \mathrm{nA}$ and no voltage offset was used. Nearly all of the minor peaks are attributable to actinides or simple molecular ions. Thorium is again seen at a few parts per million of the clay, as it was seen previously in the Pu-clay [7]. A mass spectrum of the carbon substrate over the same mass range and with the same beam conditions showed no discrete peaks and only occasional counts from detector background.

The SIMS measurement efficiencies for $U$ and $\mathrm{Pu}$ were determined by sputtering individual spheres while monitoring the five major actinide peaks in a peak-hopping mode. With a beam current of $20 \mathrm{nA}$, the signals decreased by two decades in about $15 \mathrm{~min}$ (Figure 7). The total counts of ${ }^{238} \mathrm{U}$ and ${ }^{239} \mathrm{Pu}$ were corrected for duty cycle factors, isotopic abundances, and quantum efficiency of the electron multiplier detector. The latter was calibrated on a pure uranium oxide particle by comparing the measured electron multiplier and Faraday cup count rates in the range of

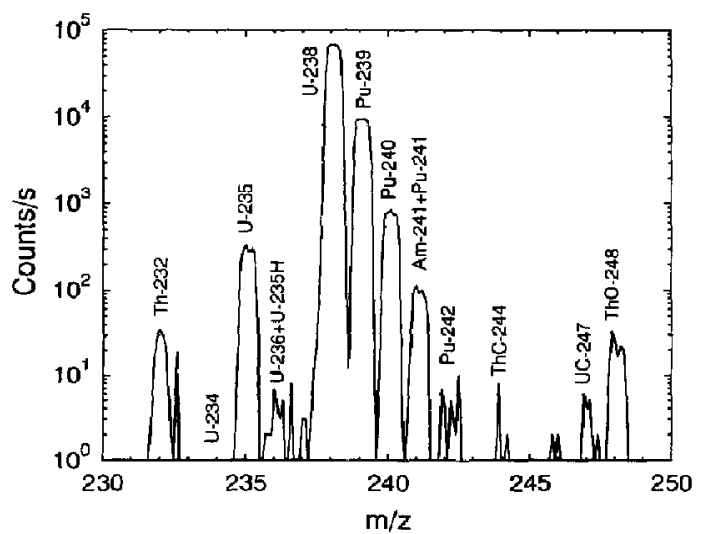

Figure 6. SIMS spectrum of the actinide mass region from a single U-Pu-clay microsphere.

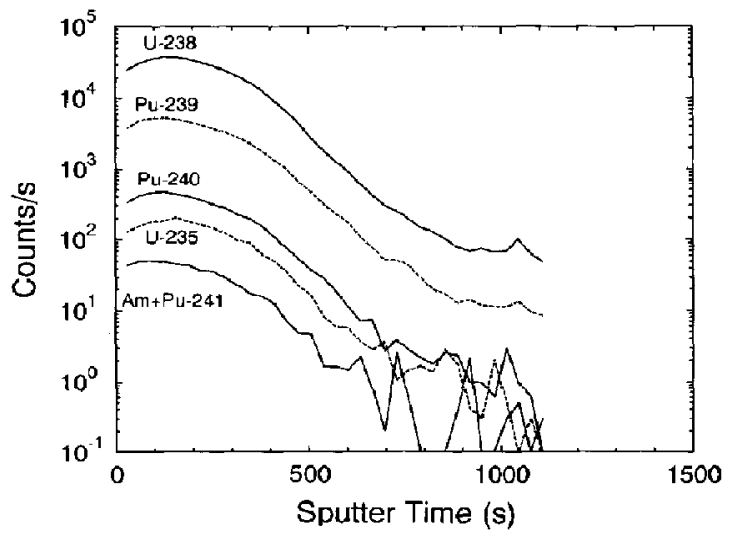

Figure 7. Time variation of the sputtered actinide ion signals from a single $\mathrm{U}-\mathrm{Pu}$-clay microsphere.

$10^{6} \mathrm{~s}^{-1}$. The quantum efficiency was found to be $63 \%$ for $\mathrm{U}^{+}$, and the same value was assumed for $\mathrm{Pu}^{+}$. The corrected ion counts for $\mathrm{U}$ and $\mathrm{Pu}$ were ratioed to the calculated number of atoms in a sphere of mean diameter.

The SIMS efficiencies for $U$ and $P u$ were determined on nine spheres. The average efficiencies were $2.9 \pm$ $0.3 \%$ ( $1 \sigma$ standard deviation) for $U$ and $8.5 \pm 0.8 \%$ for $\mathrm{Pu}$. The remarkably high efficiency for $\mathrm{Pu}$ is similar to that found for the Pu-clay $(8.4,8.4$, and $22 \%$ for three spheres [7]). The Pu:U relative sensitivity factor of 2.9 is consistent with the value of about 3.3 that was found in the Pu-clay, based on the decay products ${ }^{235} \mathrm{U}$ and ${ }^{236} \mathrm{U}$.

The isotopic ratios ${ }^{235} \mathrm{U}:{ }^{238} \mathrm{U},{ }^{240} \mathrm{Pu}:{ }^{239} \mathrm{Pu}$, and ${ }^{241}(\mathrm{Am}+\mathrm{Pu}):{ }^{239} \mathrm{Pu}$ were measured on six spheres. Measurements of the minor isotopes ${ }^{234} \mathrm{U},{ }^{236} \mathrm{U}$, and ${ }^{242} \mathrm{Pu}$ were not attempted because their count rates would be less than $1 \mathrm{~s}^{-1}$ for the analytical conditions used. Based on the calculated numbers of atoms in a microsphere and the SIMS efficiencies, 1000 ions of these minor isotopes would be produced at most, and the number detected would be further reduced by duty cycle factors. For "the isotopic measurements, the beam current was reduced to $10 \mathrm{nA}$ so the signals would not decay as rapidly as for the efficiency measurements. The sample chamber cold trap was filled with liquid nitrogen to trap residual water vapor and thus minimize the formation of hydride ions.

Typically 20 cycles of data were collected for the ratio measurements. During the first 10 cycles, the actinide signals were generally increasing and the U:Pu ratio was changing. Steady-state sputtering conditions were usually established after 10 data cycles. Therefore, only the last 10 cycles were used for the ratio calculations. A correction was made for the signal decay over this time period. A mass fractionation factor of $0.58 \pm 0.06 \% \mathrm{u}^{-1}$ was determined for uranium from isotopic measurements on six natural uranium oxide particles. This correction factor was applied to 
both the uranium and plutonium isotopic data. The average ratios with standard deviations of the mean $\left(1 \sigma_{\mathrm{m}}\right)$ among the six clay spheres analyzed are as follows:

$$
\begin{aligned}
& 235: 238=(4.916 \pm 0.043) \times 10^{-3} \\
& 240: 239=(8.694 \pm 0.040) \times 10^{-2} \\
& 241: 239=(9.590 \pm 0.110) \times 10^{-3}
\end{aligned}
$$

The 235:238 and 240:239 values are both within $2 \sigma_{\mathrm{m}}$ of the expected values of $4.960 \times 10^{-3}$ (includes 40 ppm excess from Pu decay) and $8.629 \times 10^{-2}$, respectively. Uranium hydride ions would have the largest contribution at $m / z=239$, causing the $240: 239$ ratio to be biased low. Because this is not the case, we conclude that $\mathrm{UH}^{+}: \mathrm{U}^{+}<10^{-3}$. Plutonium hydride ions would bias the $240: 239$ ratio high. Although not statistically significant, the $240 / 239$ ratio is in excess of the expected value by $6 \times 10^{-4}$. This value sets an upper limit on the $\mathrm{PuH}^{+}: \mathrm{Pu}^{+}$ratio.

The 241:239 ratio is far in excess of the expected value for $\mathrm{Pu}$ in CRM 138 of $1.9 \times 10^{-3}$ (January 1993) because of the ${ }^{241} \mathrm{Am}$ contribution. The calculated ${ }^{241} \mathrm{Am}:{ }^{239} \mathrm{Pu}$ ratio at a $\mathrm{Pu}$ age of $30.5 \mathrm{yr}$ (July 1962 to January 1993) is $6.3 \times 10^{-3}$. By comparing the excess count ratio with the expected Am ratio, a relative sensitivity factor of 1.2 for $\mathrm{Am}-\mathrm{Pu}$ is derived. This is lower than the value of 1.5 derived previously by using the same procedure for the $\mathrm{Pu}$-clay. The difference is not understood, but it is possible that different ion exchange conditions for preparation of the $\mathrm{U}-\mathrm{Pu}$-clay resulted in some loss of Am relative to the Pu-clay. Note also that there was an unusually low $\mathrm{Np}$ level detected in the earlier material, and that there are differences in $\mathrm{Na}$ concentrations for the two cases.

The interference of ${ }^{241} \mathrm{Am}$ and ${ }^{238} \mathrm{U}$ with the corresponding $\mathrm{Pu}$ isobars was not experienced with TIMS because each element evaporated at a different temperature.

\section{Conclusion}

A particulate standard, consisting of aluminosilicate microspheres loaded with minor concentrations of isotopically certified uranium and plutonium, has been produced. Individual spheres can be isolated from their slide mounts to serve as microstandards for calibration of isotope-ratio mass spectrometers, including both TIMS and SIMS. The spheres can also serve as an elemental standard for calibration of SIMS and analytical electron microprobe instruments. The microspheres are useful as instrument efficiency standards because they can be totally consumed in a short time.

\section{Acknowledgments}

We are grateful to other PNL staff members for their expert assistance. Gerald J. Powers put the uranium isotopic standard into solution. Linda $G$. Smith provided technical assistance in particle generation. Roy R. Adee performed the particle size and shape measurements on the scanning electron microscope. Rose Ann $M$. Graves mounted individual particles for $x$-ray, electron microprobe, and mass spectrometer analyses. Harvey Tenny performed the elemental analyses on the electron microprobe and did additional size measurements. Lee A. Bond performed the isotopic analyses on the PNL triple-sector mass spectrometer. James M. Kelley assisted with helpful discussions. We also thank George J. Newton of the Inhalation Toxicology Research Institute, Albuquerque, for many helpful discussions. PNL is operated for the U.S. Department of Energy by Battelle Memorial Institute under Contract DE-AC06-76RLO 1830.

\section{References}

1. Simons, D. 5. J. Trace Microprobe Tech. 1986, 4, 185-195.

2. Stoffel(s), J. J.; Cannon, W. C.; Robertson, D. M. J. Am. Soc. Mass Spectrom: 1991, 2, 81-84.

3. Raabe, O. G.; Kanapilly, G. M.; Newton, G. J. In Inhaled Particles III; Walton, W. H., Ed.; Unwin Brothers: Old Woking, England, 1971; pp 3-17.

4. Newton, G. J.; Kanapilly, G. M.; Boecker, B. B.; Raabe, O. G. In Genteration of Aerosols and Facilities for Exposure Experiments; Willeke, K., Ed,; Ann Arbor Science Publishers: Ann Arbor, 1980; pp 399-425.

5. Kotrappa, P,; Moss, O. R. Health Physics 1971, 21, 531-535.

6. Kirby, H. W.; Sheehan, W. E. Nucl. Instrum. Methods 1984, $223,356-359$.

7. Simons, D. S. In SIMS VIII: Proceedings of the Eighth International Conference on Secondary lon Mass Spectrometry; Benninghoven, A.; Janssen, K. T. F.; Tümpner, I.; Werner, H. W., Eds.; John Wiley \& Sons: Chichester, England, 1992, pP 715-718.

8. Lagergren, C. R.; Stoffels, J. J. Int. J. Mass Spectrom. Ion Phys. 1970, 3, 429-438.

9. Kelley, J. M.; Robertson, D. M. Anal. Chem. 1985, 57, 124-130. 